\title{
Stress Testing: A Low Cost Alternative for Burn-in
}

\author{
Rabindra Roy, Kaushik Roy†, and Abhijit Chatterjeeł \\ NEC USA, Purdue University†, Georgia Inst. of Tech. $\ddagger$ \\ $U S A$
}

\begin{abstract}
With the increasing complexity of VLSI circuits, reliability has been of great concern to the semiconductor and system manufacturers. The existing test methodologies, e.g. application of test set for stuck-at faults, are good for ensuring that the circuit is operational at the time of testing. Due to imperfections in manufacturing process, some circuits have infant mortality problems, which implies that the circuits have a high probability of failure during the early part of its useful life. Burn-in, which stresses the circuit under controlled environments, has been traditionally used to weed out circuits with infant mortality problems. However, some practical considerations such as cost or unavailability of burn-in ovens may limit the use of burn-in. If the cost of burn-in is prohibitive, then there are no known alternatives for improving reliability of the shipped circuits. In this paper we propose a stress testing method which can provide an attractive low-cost alternative to burnin. Stress testing can also be applied at the die level to generate Known Good Dies (KGD) for Multi-Chip Modules (MCMs). The test methodology can generate electrical or current stress in a circuit achieved using any of the following methods, namely (1) by generating high current density in the all parts of circuit, or (2) by generating high current density in selected parts of the circuit, resulting in hot spots and thermal gradient. Since stress testing should achieve high fault coverage to cover functional faults as well, one clever idea is to use a stuck-at fault test set, and modify it to perform the stress test.
\end{abstract}

Testing, Burn-in, Power dissipation

\section{Keywords}

\section{INTRODUCTION}

Electronic integrated circuits (ICs) are increasingly found in new application domains, for example, medical and automotive applications. Such use is possible due to the miniaturization through advancement of technology, resulting in higher performance, lower cost, and increased complexity of ICs. Application domains, in which a failure can have catastrophic effects, require circuits 


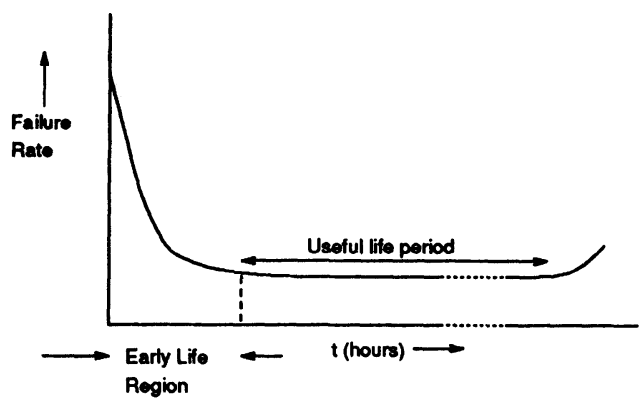

Figure 1 Bathtub curve for failure rate vs. time

with higher quality and reliability. Even the best design and fabrication techniques can propagate some reliability and/or device quality problems with the end product. In order to ensure product quality, testing is done at various levels during the design process and circuits failing the test are discarded. It has been observed that after the chip is fabricated, the failure rate follows the bath tub curve as shown in Figure 1. During the infant mortality period or the early life region, the failure rate is much higher compared to that during the useful life region. This phenomenon occurs because in a large sample of ICs produced, there are some units which have some borderline manufacturing defects, such that their life is relatively short compared to other units of the same sample. These units are said to have infant mortality problems. From a given sample, if all the units with such infant mortality problems are sorted out, then the reliability of the remaining units of the sample will be higher. Units with infant mortality problems often have latent defects which are not noticed during normal testing of the VLSI circuits. Such defects can be activated by stressing the circuit with higher current densities and temperature. It has been observed in [7] that larger current densities can stress defects such as epitaxial and crystal imperfections, metallization, oxide, and junction anomalies. Many of these defects require localized current stress to provide activation for the associated failure mechanism.

\subsection{Burn-in vs. Stress Testing}

Burn-in is a well-known technique that can provide the stressing of the circuit needed for weeding out circuits with infant mortality problems [6]. However, burn-in is a very expensive procedure. It requires special ovens to maintain a high temperature. Among the three known techniques of burn-in, static, dynamic, and monitored, monitored burn-in is most effective in detecting reliability defects. It requires application of test vectors during burn-in. The number of available input/output lines in a burn-in oven increases the price of burn-in ovens significantly, and hence, monitored burn-in can be fairly expensive. Currently, there are no known low-cost alternative to burn-in. 
In this paper we present a test methodology which can potentially weed out some of the latent defects which are activated by higher current densities or localized current stress while achieving a high fault coverage for the stuck-at faults. Stress testing is a new proposed method which involves running stress test patterns on a circuit for an extended period of time, such that units with infant mortality problems fail. The stress test patterns should be such that they activate all parts of the circuit to create high current densities. We have developed a method where we use an already existing test set and reorder it to achieve our goals. If stress testing needs to be performed for a long period of time, then the same test set can be repeated.

The stress test methodology can also find application in testing of dies for Multi-Chip Modules (MCMs). To achieve high yield for the modules, it is essential to use the Known Good Dies (KGD) [2]. Several researchers [3,4] have suggested that wafer-level and die-level stress testing should be used to achieve the KGDs. Hence, in the absence of die-level burn-in, the stress testing techniques can be used to determine the known good dies. The testing technique can also be used during monitored burn-in $[5,6,7]$ to enhance the localized current stress in parts of the circuit.

\subsection{Current Increase in CMOS Circuits}

For functional verification of a circuit, test vectors are generated targeting a particular fault model such as the stuck-at faults. Stuck-at faults have been traditionally used to model manufacturing defects [1]. To achieve high defect coverage, the test vectors should be such that $100 \%$ fault coverage is achieved for the list of faults under consideration. In our test methodology, we reorder the input stuck-at test vectors such that a high current density is achieved in a predefined portion of the circuit, while testing for stuck-at faults. In CMOS circuits, majority of the current flow is due to the signal switching. Hence, by reordering the stuck-at test vectors we try to increase the switching activity, and hence the current, in certain specified regions of the circuit.

The estimation of average current due to switching activity (average number of transitions per unit time) has been considered in $[10,11,12]$ where the switching activities at the internal nodes of a circuit are estimated using probabilistic techniques, based on input signal probabilities (probability of having a logic ONE) and switching activities. Experiments suggests that average switching current can be accurately estimated using the signal activity measure at the internal nodes. Recently Chou et. al. [8] considered scheduling of tests so as to minimize power dissipation. They provide theoretical results for the power constrained test scheduling problem. The objective was to minimize total test length subject to the power constraint. In this paper we present a methodology to selectively control current flow in sections of the circuit while testing for stuck-at faults. 


\section{PRELIMINARIES}

In this section we describe a model for estimating the average current in CMOS circuits. The current drawn by a CMOS circuit is intimately related to the power dissipation. We will first estimate the average power dissipation of a CMOS multi-level combinational logic circuit due to signal activity. Power Dissipation in CMOS: The three sources of power dissipation in CMOS circuits are due to leakage current, short-circuit current, and switching current associated with charging or discharging of load capacitances. The latter component accounts for majority of the power dissipation in CMOS and will only be considered in our discussions. The capacitances internal to a logic gate are assumed to be small and are neglected in our analysis. The average power dissipation in a multi-level logic circuit is given by

$$
P_{a v g}=\frac{1}{2} V_{d d}^{2} \sum_{i=1}^{n} A_{i} C_{i}
$$

where $V_{d d}$ is the supply voltage and is constant, $C_{i}$ is the capacitance associated with node $i$ of the circuit, and $A_{i}$ is the activity at node $i . A_{i}$ is equal to the average number of transitions per unit time. The summation is taken over all the $n$ nodes of the circuit. From Equation 1 it is clear that the average current $I_{d d}$ drawn from the supply voltage due to the switching component of power is equal to $I_{d d}=\frac{1}{2} V_{d d} \sum_{i=1}^{n} A_{i} C_{i}$. Hence, both average power dissipation and switching current are proportional to the weighted switching activity given by $\sum_{i=1}^{n} A_{i} C_{i}$.

For a gate level description of the circuit, the load capacitance of each gate can be approximated by the fanout times the transistor input capacitance. However, estimation of signal activity at the nodes is not trivial. All published methods of estimation of signal activity involves estimation of signal probability, which is the probability of a signal taking a logic value of ONE. If the primary input signal probabilities and activities are known, probabilistic or simulation based techniques $(10,11,12,13)$ can be used to estimate the activities at internal nodes of a circuit.

In this paper, the exact input stimulus to the circuit under the test mode is known. Hence, a simulation-based technique can be used to obtain the actual activity at the internal nodes of a circuit. It should be observed that if accurate delay models for the logic gates and interconnects are available, then this technique will be able to accurately determine the spurious transitions at the internal nodes of the circuits. The spurious transitions causes switching of internal nodes and hence, dissipates power. The details of the power estimation technique during input reordering is given in Section ??. 
The maximum current is important when the circuit has to be stressed to its limit. From Equation 1 we can observe that for a given set of stuck-at test vectors $V$, the condition for maximum current is given by

$\operatorname{maximize}\left(\sum_{i=1}^{n} T_{i}^{v_{k}, v_{l}} C_{i}\right) ; \quad v_{k}, v_{l} \in V$

where $T_{i}^{v_{k}, v_{l}}$ is given by

$T_{i}^{v_{k}, v_{l}}= \begin{cases}0 & \text { if } v_{k} \text { followed by } v_{l} \text { produces no transition at node } i \\ 1 & \text { if } v_{k} \text { followed by } v_{l} \text { produces a transition at node } i\end{cases}$

$v_{k}$ and $v_{l}$ represents two stuck-at test vectors from set $V$. The term $T_{i}^{v_{k}, v_{l}}$ is similar to activity defined in Equation 1. If one considers the nodes of a circuit to have finite delays, then glitches can occur. For such transitions, the range of values of $T_{i}^{v_{k}, v_{l}}$ can be larger than 1 based on whether no transition or a functional or hazardous (static or dynamic hazard) transition is present at node $i$.

Thermal Modeling of ICs: Most of the electrical energy consumed by an IC eventually appears as heat. An increase in device temperature can be achieved by dissipating more heat through increased activity. The accelerated aging of an IC can be expressed as an exponential function of the junction temperature as:

$t_{A}=t_{0} \exp \left[\frac{E}{k}\left(\frac{1}{T_{0}}-\frac{1}{T_{j}}\right)\right]$

where

$t_{\boldsymbol{A}}=$ lifetime at elevated junction $\left(T_{j}\right)$ temperature

$t_{0}=$ normal lifetime at normal junction $\left(T_{0}\right)$ temperature

$E=$ activation energy (ev)

$k=$ Boltzmann's constant $\left(8.617 \times 10^{-5} \mathrm{ev} / \mathrm{K}\right)$

For a certain process, the lifetime of an IC could be decreased by a factor of 2 for every $10^{\circ} \mathrm{C}$ increase in temperature [18]. Units with infant mortality problems have a low lifetime, which can be shortened further by increasing temperature through stress testing, thereby weeding them out with a reasonably long stress testing time. 


\section{GRAPH-BASED FORMULATION OF THE PROBLEM}

The problem of reordering test vectors to achieve the required current stress can be formulated as a graph traversal problem [14] for efficient solution. To better understand the procedure, let us consider the following definition.

Definition 1 (Hamiltonian Path) A graph $G(V, E, W)$ consisting of a set of nodes $V$, a set of edges $E$ and a set of weights $W$ (each edge associated with a weight) is said to have a Hamiltonian path if there is an ordering of nodes $\left\langle v_{1}, v_{2}, \ldots, v_{m}\right\rangle$ of $G$, where $m=|V|$, such that, $\left\langle v_{i}, v_{i+1}\right\rangle \in E$ for all $i, 1 \leq i<m$. The ordering $\left\langle v_{1}, v_{2}, \ldots, v_{m}\right\rangle$ represents a Hamiltonian path of graph $G$. The cost of the Hamiltonian path is given by the summation of weights associated with each edge of the Hamiltonian path.

Let us consider a set of stuck-at test vectors $V$ of size $m$ for a combinational circuit $\mathcal{C}$. In order to determine the switching activity due to the application of the test vectors we consider a graph $G(V, E, W)$ whose nodes are given by the set $V$. The cardinality of set $V$ is $m$, which is the number of test vectors to be applied to circuit $\mathcal{C}$ under test. $E$ is the set of edges in the graph, while $W$ represents the set of weights associated with each edge. For example, an edge connecting nodes $v_{i} \in V$ and $v_{j} \in V$ is given by $e_{i j} \in E$ and represents that test vector $v_{j}$ is applied to the circuit after application of vector $v_{i}$. Each edge is also associated with a weight $w_{i j} \in W$. Let us also assume that the graph is fully connected, i.e. there exists an edge between every pair of vertices $v_{i}$ and $v_{j},(i \neq j)$. Such graphs are referred to as cliques. The weight $w_{i j}$ associated with each edge $e_{i j}$ is given by the switching activity over all circuit nodes due to application of test vectors $v_{i}$ followed $v_{j}$. Hence, $w_{i j}$ is proportional to the current associated with the application of input vectors $v_{i}$ and $v_{j}$, respectively, and is given by

$w_{i j}=\sum_{k=1}^{n} T_{k}^{v_{i}, v_{j}} C_{k}$

where $C_{k}$ is the capacitive loading associated with node $k$ of circuit $\mathcal{C}$ having $n$ number of nodes. Assuming the transistor input capacitance to be constant for all transistors of a design, $C_{k}$ can be approximated by the number of fanouts at node $k$.

For graph $G(V, E, W)$, each node represents a test vector $v_{k}$ for the CMOS circuit $\mathcal{C}$, and each edge $e_{i j}$ represents the current flow or power dissipation when vectors $v_{j}$ is applied after the application of vector $v_{i}$. Hence, a Hamiltonian path of the graph $G$ starting from node $v_{1}$ and ending in $v_{m}$ 


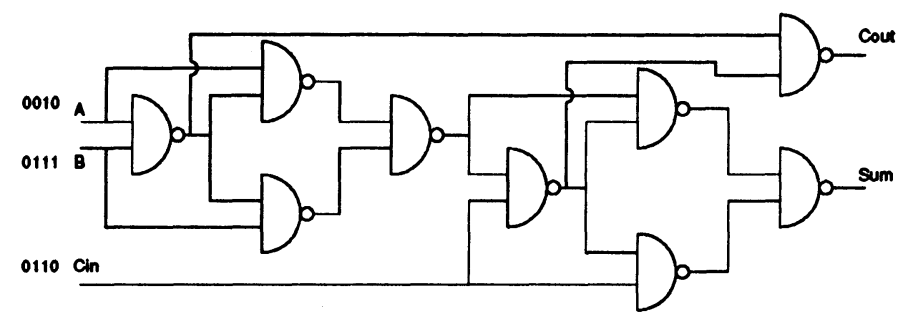

Figure 2 Full adder

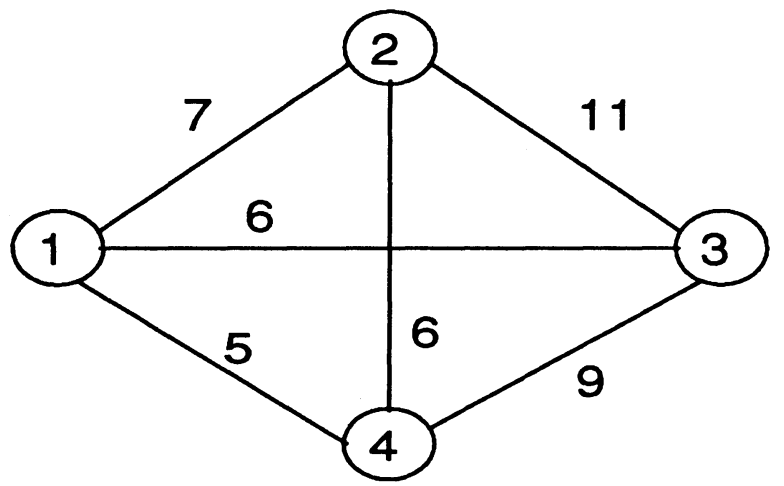

Figure 3 Graph $\mathrm{G}$ for the 4 test vectors of the circuit in Figure 2

$\left\langle v_{1}, v_{2}, v_{3}, \ldots, v_{m}\right\rangle$ represents an ordering of test vectors for which the average current is proportional to

$P=\frac{1}{m} \sum_{i=1}^{n-1} w_{i, i+1}$

This suggests that the maximum or minimum length Hamiltonian path is an ordering of test vectors which produces maximum or minimum average current, respectively. Let us consider an example. Figure 3 shows the graph $G$ for the four test vectors applied to a 1-bit full adder of Figure 2. The weight associated with each edge represents the actual simulation result of applying two test vectors to the adder circuit. For example $w_{12}$ represents the switching activity when test vector 2 is applied after application of vector 1 . The capacitance associated with each node of the full adder is approximated by the number of fanout at that node. Glitches were neglected. From Figure 3 it is clear that the maximum Hamiltonian path is $\left\langle v_{1}, v_{2}, v_{3}, v_{4}\right\rangle$. Hence, such a sequence of test vectors will produce the maximum average current. While a minimum Hamiltonian path, represented by $\left\langle v_{2}, v_{4}, v_{1}, v_{3}\right\rangle$ produces minimum average current.

The problem of determining the maximum or a minimum Hamiltonian path or a path of length close to a specified value, is similar to the traveling salesman 
problem for which there exists no known polynomial time algorithm [14]. Hence, we resort to approximate methods of solution.

\subsection{Reordering for Maximal or Minimal Activity}

The graph $G(V, E, W)$ can be represented by an $m \times m$ matrix $M$ such that the $(i, j)$ th entry $M_{i j}$ is equal to $w_{i j} . M_{i i}$ is undefined. We use a greedy algorithm to determine the reordered test vectors for maximal or minimal current stress. A very similar procedure can be used for minimizing the average current.

\section{Algorithm 1:}

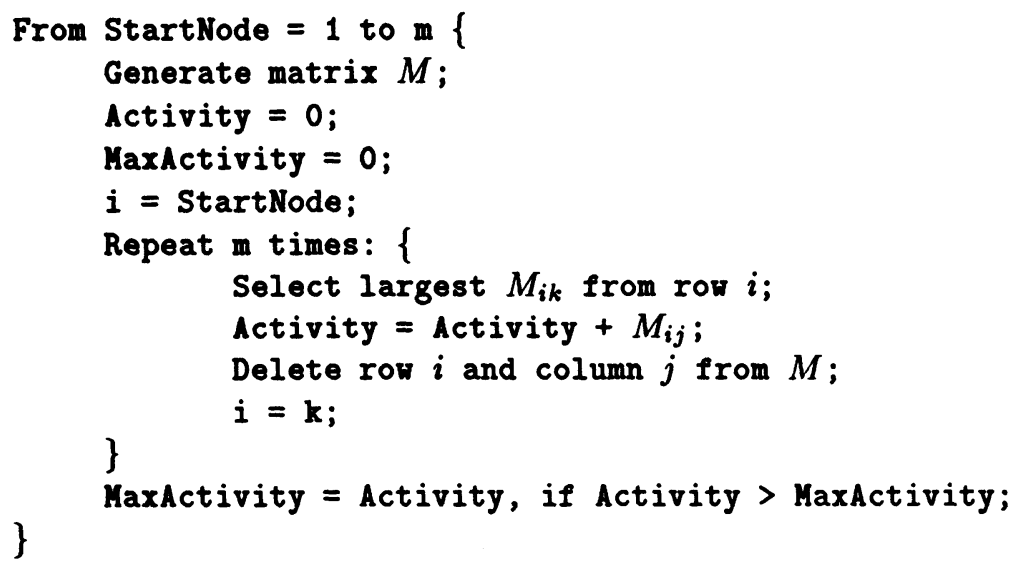

Starting from a row $r$ (test vector or node) of matrix $M$ the greedy algorithm selects the largest (smallest) entry in that row. That takes time $O(m)$. The row $(r)$ and column $(c)$ corresponding to the maximum (minimum) entry is removed from matrix $M$. Note that $c$ represents the next node visited from node $r$. Row $c$ is selected next for the above operation. The process is repeated $m$ times to determine a Hamiltonian path starting at node StartNode. The entire process is again repeated with a different starting node for the Hamiltonian path. Hence, the algorithm takes $O\left(\mathrm{~m}^{3}\right)$ time.

\subsection{Reordering for Desired Activity}

In order to achieve a desired circuit activity by test reordering, a procedure similar to the one described above can be used. If the desired activity is $D$, then we define the average activity per node, $D_{a}=D / m$, where $m$ is the number of nodes of graph $G(V, E, W)$. Instead of selecting the largest $M_{i k}$, as shown in Algorithm 1, the value of $M_{i k}$, which gives the closest running 
average to $D_{a}$ is chosen. This approach leads to reordering such that activity is very close to the desired value. In case the desired value of activity is higher or lower than the maximal or minimal value as obtained in Section 3.1, the algorithm will return the maximal or the minimal value, respectively. A sketch of the algorithm is given below.

\section{Algorithm 2:}

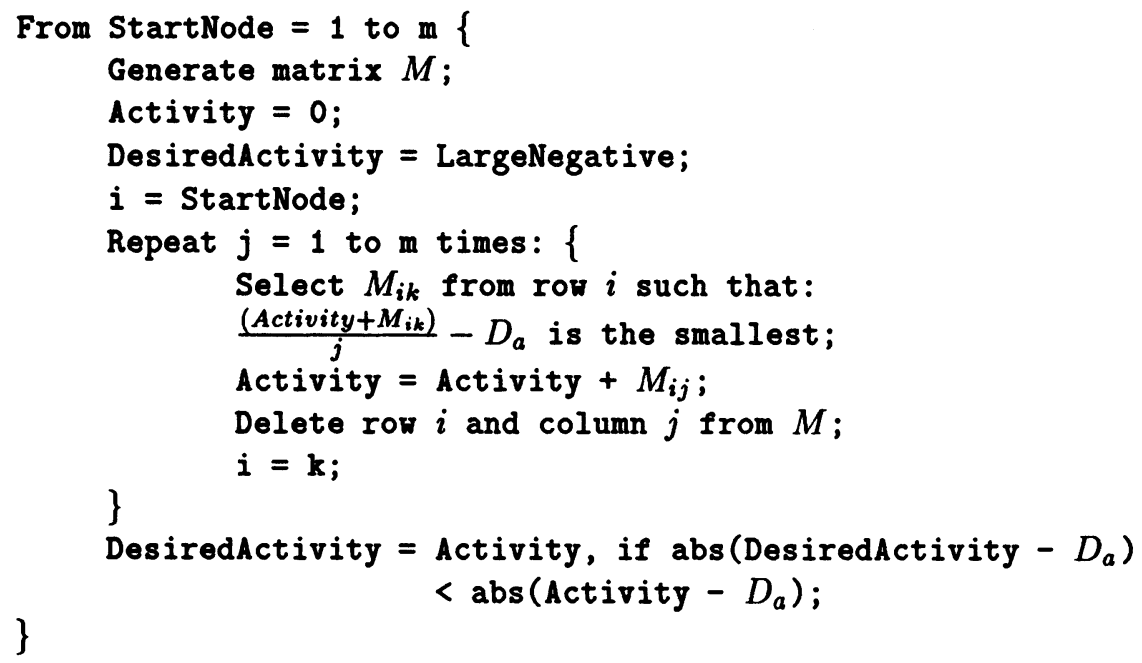

\subsection{Reordering for Localized Current Stress}

Hnatek in [7] observed that defects such as epitaxial and crystal imperfections, metallization, oxide, or junctional anomalies may require localized current stresses for the activation of the failure mechanism. In this subsection we will consider reordering of stuck-at test vectors to achieve such a stress.

Let us first consider the case when a chip is partitioned into two parts: Part and Part $_{2}$. In order to have a large current gradient between Part $_{1}$ and Part the activity (weighted by capacitance) in one part should be maximized while the activity (weighted by capacitance) in the other part should be minimized. The activities weighted by the corresponding capacitances in the two parts can be easily determined by applying a test vector $v_{i}$ followed by vector $v_{j}$ and by noting the number of nodes undergoing transition. Hence, one can construct two completely connected graphs $G_{1}\left(V, E, W^{1}\right)$ and $G_{2}\left(V, E, W^{2}\right)$, where the respective edges $e_{i j}^{1}$ and $e_{i j}^{2}$ between nodes $v_{i}$ and $v_{j}\left(v_{i}, v_{j} \in V\right)$ are weighted by the activity from the corresponding parts. It should be noted that the number of vertices in each graph $G_{1}$ and $G_{2}$ is equal to the number 
of test vectors. The difference between the weights associated with edges $e_{i j}^{1}$ and $e_{i j}^{2}$ is equal to

$$
\Delta_{i j}=w_{i j}^{1}-w_{i j}^{2}
$$

which signifies the difference in activity between the two parts, when two test vectors $v_{i}$ and $v_{j}$ are applied in sequence. Now, it is possible to construct a completely connected graph $G_{\delta}\left(V, E, W^{\delta}\right)$ such that each edge weight $w_{i j}^{\delta}=\Delta_{i j}$. Using the algorithms described above, it is possible to find a Hamiltonian path which is maximal, minimal, or of a specified length. The maximal Hamiltonian path corresponds to the ordering of test vectors which produces the maximal current gradient between Part $_{1}$ and Part 2 , while the specified weight produces the specified current gradient between the parts.

Let us consider the more general case when a circuit is partitioned into $p$ parts. It is possible to generate graphs $G_{1}\left(V, E, W^{1}\right), \ldots, G_{p}\left(V, E, W^{p}\right)$ for each of the parts such that the weight $w_{i j}^{k}$ associated with edge $e_{i j}^{k}$ for part Part $_{k}$ is equal to the activity in the part due to the application of test vectors $v_{i}$ followed by $v_{j}$. Each part $k$ can be associated with a stress weight $s_{k}$ which specifies the relative stress that each of the parts are required to experience. For example, in case of the two-way partitioning as described above, $s_{1}$ and $s_{2}$ should be 1 and -1 respectively, to achieve maximum current gradient between the parts. The edge weights in each graph $G_{k}\left(V, E, W^{k}\right)$ is multiplied by $s_{k}$ to include the relative stress weighting factor into account. The new edge weight $n w_{i j}^{k}$ is given by

$$
n w_{i j}^{k}=w_{i j}^{k} * s_{k}
$$

A modified graph $G_{\delta}\left(V, E, W^{\delta}\right)$ is constructed such that weight of each edge is given by

$w_{i j}^{\delta}=w_{i j}^{1}+w_{i j}^{2}+\ldots+w_{i j}^{p}$

The maximum Hamiltonian path in graph $G_{\delta}\left(V, E, W^{\delta}\right)$ produces the near optimal stress across the different parts.

Circuit partitioning for stress testing can only be done after the chip layout. From the chip layout, the reliability engineer can determine the sections of the chip across which high current gradient is required. Based on such information, it is possible to determine the logic gates in each part. We have taken a layout of a $4 \mathrm{X} 4$ multiplier and performed such stress testing by partitioning it into three parts. 


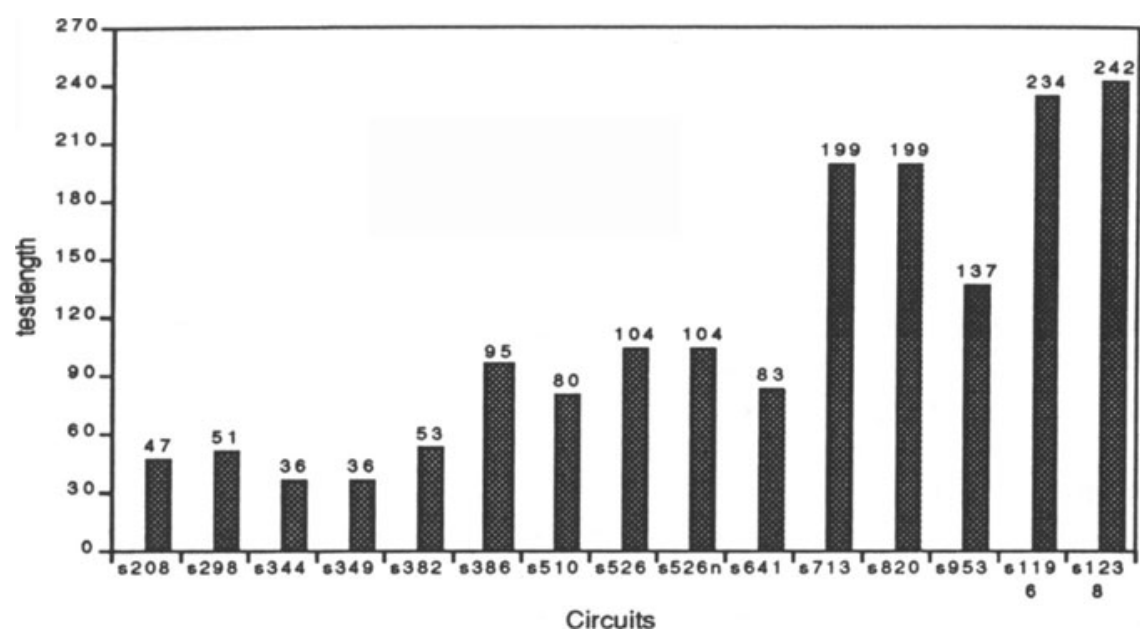

Figure 4 Test lengths for $100 \%$ test efficiency

\section{IMPLEMENTATION AND RESULTS}

The algorithms described earlier have been implemented in C on SUN workstations. The circuits used for experimentation are full-scan version of a subset of the ISCAS-89 [17] benchmark suite, which are used extensively in the VLSI CAD (Computer Aided Design) research for evaluating any new technique in testing and synthesis. The circuits were run through an automatic stuck-at test generator, TRAN [16], to get test patterns with $100 \%$ test efficiency. These test patterns were the target for reordering such that the required current stress could be achieved. Figure 4 shows the test lengths (or the number of test vectors) of the circuits for $100 \%$ test efficiency.

Figure 5 graphically shows the comparison between minimum and maximum current drawn by a circuit by reordering of the input patterns. It is interesting to note that current requirements during testing can be changed by more than $300 \%$ by reordering the test vectors.

Table 1 shows the performance of our algorithm when a desired activity is required during testing of a VLSI chip. The results based on Algorithm 2 shows that it is possible to control the circuit activity within $5 \%$ of the desired value.

In order to see the effect of reordering test vectors to create electrical stress across different partitions, we considered a multiplier circuit. Figure 6 shows the layout of a $4 \times 4$ multiplier. Test vectors were generated for the multiplier using TRAN [16] for $100 \%$ coverage of stuck-at faults. Table 2 shows the results of reordering the test vectors to create the maximum stress gradient between different partitions of the layout of Figure 6 . The activity ratio refers to the ratio of activity in the two partitions under considerations. Results on three different partitions have been presented. Results show that a large 


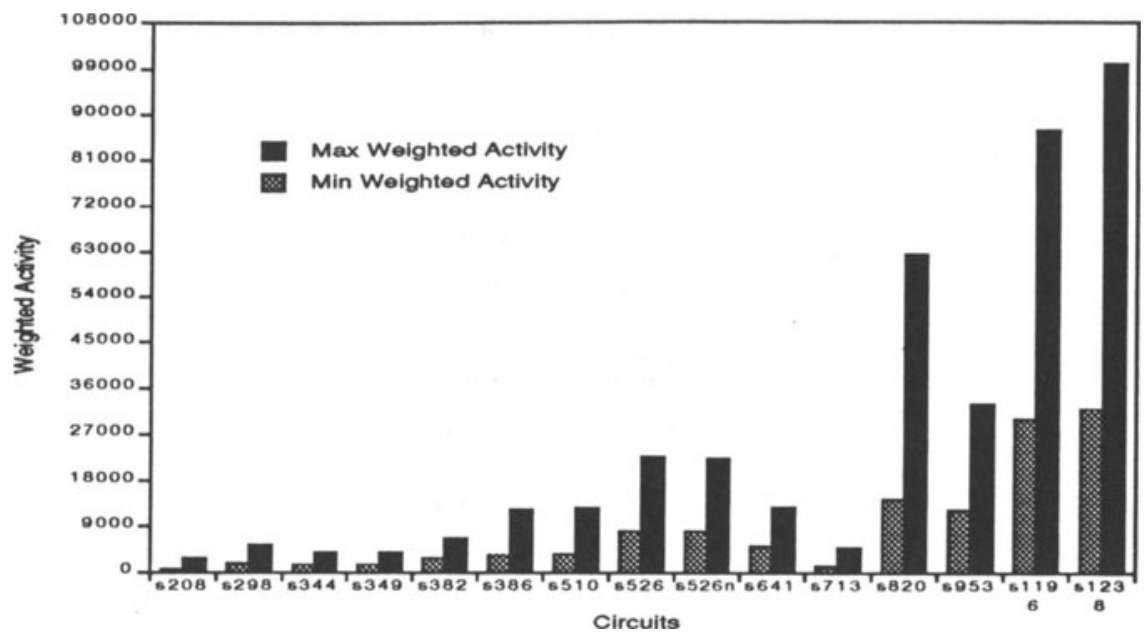

Figure 5 Minimum and maximum current drawn by circuits

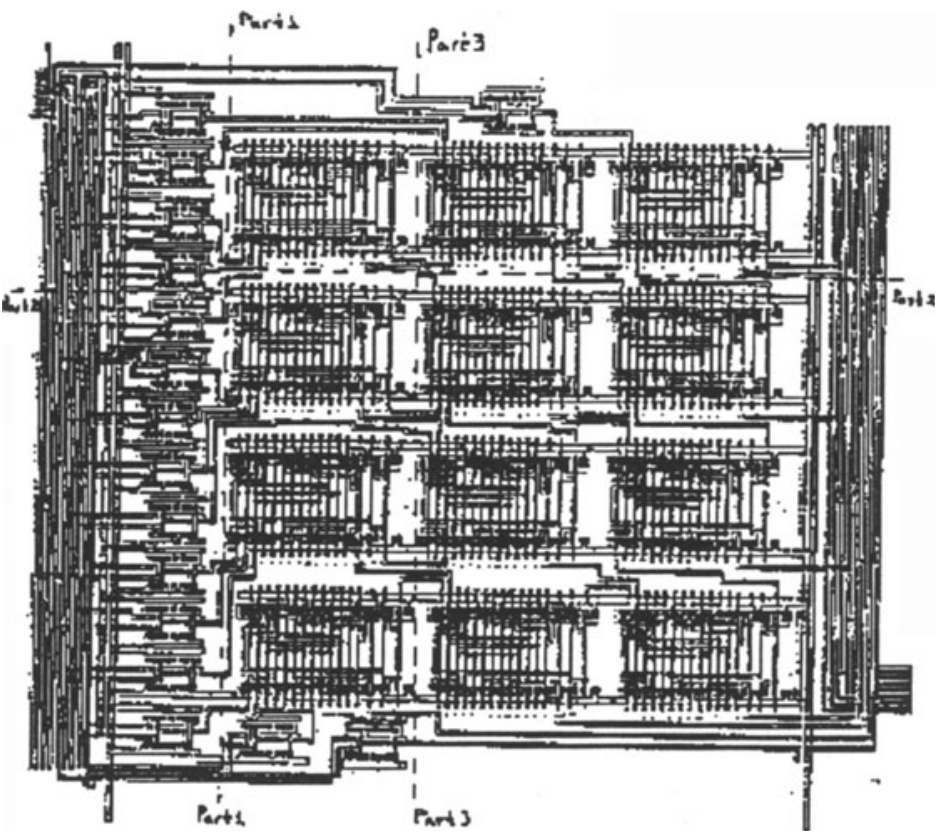

Figure 6 A 4x4 multiplier layout 
Table 1 Comparison of desired and actual power values

\begin{tabular}{lcc}
\hline $\begin{array}{l}\text { Design } \\
\text { Name }\end{array}$ & $\begin{array}{c}\text { Desired } \\
\text { Activity }\end{array}$ & $\begin{array}{c}\text { Actual } \\
\text { Activity }\end{array}$ \\
\hline s208 & 800 & 861 \\
& 1700 & 1699 \\
& 2100 & 2082 \\
\hline s298 & 3000 & 3001 \\
& 4000 & 3965 \\
& 5000 & 4812 \\
\hline s510 & 5000 & 5099 \\
& 7000 & 7000 \\
& 10000 & 9951 \\
\hline
\end{tabular}

Table 2 Results for the multiplier example

\begin{tabular}{lc}
\hline Partition & Activity ratio \\
\hline Part1 & 10.69 \\
Part2 & 9.10 \\
Part3 & 5.12 \\
\hline
\end{tabular}

activity gradient can be produced based on the stuck-at test vectors of the circuit.

\section{DISCUSSIONS AND FUTURE DIRECTIONS}

In this paper we have presented a novel approach to reordering of stuck-at test vectors to create electrical stress during functional testing of the circuit. Such electrical stress can activate some latent defects and can weed out defects such as epitaxial and crystal imperfections, metallization, oxide, and junction anomalies. The technique can also be used to obtain the Known Good Dies for MCMs when die-level burn-in is not available. The methodology is simple, and utilizes existing test patterns for any good fault model, thus rendering itself to be a low-cost alternative to well established burn-in technique. The proposed method should be investigated further to answer some practical questions. What should be the duration of stress test? What kind of circuits are more 
amenable to stress test? Also, what range of improvement in reliability can be expected by application of stress test?

\section{REFERENCES}

[1] J. Abraham and W. Fuchs, "Fault and Error Models for VLSI," Proc. of IEEE, May 1986, pp. 639-654.

[2] D. C. Keezer, "Known Good Die for MCMs: Enabling Technologies," Proc. of Intl. Test Conf., 1993, pp. 266.

[3] W. Daum and W. Burdick, Jr., "Minimal IC Pretest Requirements for Multichip Modules," IEEE Trans. on Components, Packaging, and Manufacturing Technology - Part B, Aug. 1994, pp. 277-282.

[4] Y. Zorian, "A structured Testability Approach for Multi-Chip Modules Based on BIST and Boundary-Scan," IEEE Trans. on Components, Packaging, and Manufacturing Technology - Part B, Aug. 1994, pp. 283-290.

[5] M. Campbell, "Monitored Burn-In (A Case Study for In-Situ Testing and Reliability Studies)," IEEE Intl. Test Conference, 1984, pp. 518-523.

[6] A. van den Heuvel and N. Khory, "A Rational Basis for Setting Burn-In Yield Criteria," IEEE Intl. Test Conference, 1984, pp. 524-530.

[7] E. Hnatek, "Thoughts on VLSI Burn-In," IEEE Intl. Test Conference, 1984, pp. 531-535.

[8] R. Chou, K. Saluja, and V.D. Agrawal, "Power Constraint Scheduling of Tests," IEEE Intl. Conf. on VLSI Design, 1994, pp. 271-274.

[9] A. Chandrakashan, S. Sheng, and R. Brodersen, "Low Power CMOS Digital Design," IEEE Journal on Solid-State Circuits, Vol. 27, No. 4, Apr. 1992, pp. 473-484.

[10] K. Roy and S. Prasad, "Circuit Activity Based Logic Synthesis for Low Power Reliable Operations," IEEE Trans. on VLSI Systems, Dec. 1993, pp. 503-513.

[11] F.N. Najm, "Transition Density, A Stochastic Measure of Activity in Digital Circuits," ACM/IEEE Design Automation Conf., 1991, pp. 644649 .

[12] R. Burch, F. Najm, P. Yang, and T. Trick, "A Monte Carlo Approach for Power Estimation," IEEE Trans. on VLSI Systems, Mar. 1993, pp. 63-71.

[13] A. Ghosh, S. Devadas, K. Keutzer, and J. White, "Estimation of Average Switching Activity in Combinational and Sequential Circuits," ACM/IEEE Design Automation Conf., 1992, pp. 253-259.

[14] M. Garey and D. Johnson, Computers and Intractability: $A$ Guide to the Theory of NP-Completeness, W.H. Freeman and Company, New York, NY, 1979.

[15] P. Horowitz and W. Hill, The Art of Electronics, Cambridge University Press, New York, NY, 1989.

[16] S. T. Chakradhar, V. D. Agrawal, and S. G. Rothweiler, "A Transitive Closure Algorithm for Test Generation," IEEE Trans. CAD, July 1993, pp. 1015-1028.

[17] F. Brglez, Combinational Benchmark Circuits, Intl. Symp. on Circuits and Systems (ISCAS), 1989.

[18] IDT Technologies, IDT CMOS Logic Handbook, Mountain View, CA, 1993. 